\title{
GANHO DE PESO NOS PACIENTES PORTADORES DE MIELOMENINGOCELE APÓS TRATAMENTO COM PRÓTESE VERTICAL EXPANSÍVEL DE TITÂNIO PARA COSTELA
}

\author{
WEIGHT GAIN IN PATIENTS WITH MYELOMENINGOCELE AFTER TREATMENT \\ WITH VERTICAL EXPANDABLE PROSTHETIC TITANIUM RIB
}

\section{AUMENTO DE PESO EN PACIENTES CON MIELOMENINGOCELE DESPUÉS DEL TRATAMIENTO CON LA PRÓTESIS VERTICAL EXPANSIBLE DE TITANIO PARA COSTILLA}

Rafael Maroues lelpo', Paulo Tadeu Maia Cavali², Alexander Junqueira Rossato ${ }^{3}$, Maurício Antonelli Lehoczki ${ }^{3}$, Marcelo ltalo Risso-Neto ${ }^{4}$, Guilherme Rebechi Zuiani ${ }^{4}$, IVan Guidolin Veiga ${ }^{4}$, Wagner PasoualinI ${ }^{4}$, Élcio Landim ${ }^{5}$

\begin{abstract}
RESUMO
Objetivo: Avaliar o ganho de peso e a variação do estado nutricional dos pacientes portadores de mielomeningocele torácica após o uso da prótese vertical expansível de titânio (VEPTR) como opção para tratamento sem fusão da coluna vertebral. Métodos: Estudo retrospectivo de oito pacientes (com média de idade de 5,3 anos; variando de 3,6 a 7,6 anos no momento da cirurgia inicial), portadores de mielomeningocele torácica que apresentavam escoliose, medida pelo método de Cobb, superior a $40^{\circ}$ e cifose superior a $80^{\circ}$, tratados com VEPTR pelo grupo de Escoliose da AACD de São Paulo. Foram analisados os pesos, desvio-padrão em que cada paciente se encontrava na tabela idade versus peso referência da OMS e valores angulares da cifose, no pré-operatório, pós-operatório imediato e pós-operatório tardio. Resultados: Houve ganho absoluto de peso em todos os pacientes, com média de 8,7 kg (variando de 3 a $20 \mathrm{~kg}$ ). Seis pacientes (75\%) apresentaram ganho de peso e aumento do valor do desvio padrão, traduzindo-se em aproximação de seu peso com relação à mediana considerada normal da curva idade versus peso referência após o início do tratamento com VEPTR.Conclusão: O ganho de peso absoluto observado não foi acompanhado de melhora do estado nutricional e não houve relação entre correção da cifose e ganho de peso.
\end{abstract}

Descritores: Meningomielocele; Próteses e implantes; Ganho de peso; Cifose; Coluna vertebral.

\begin{abstract}
Objective: To evaluate weight gain and change in nutritional status of patients with thoracic myelomeningocele after using the vertical expandable prosthetic titanium rib (VEPTR) as an option for treatment of the spine without fusion. Methods: We conducted a retrospective study including eight patients (mean age of 5.3 years, ranging from 3.6 to 7.6 years at the time of initial surgery), with thoracic myelomeningocele presenting with scoliosis higher than $40^{\circ}$ and kyphosis greater than $80^{\circ}$, measured by the Cobb method. All patients were surgically treated with VEPTR by the Scoliosis Group of the AACD in São Paulo. We analyzed patient weights and nutritional status by comparing the standard deviations in weight for age groups based on the WHO reference chart. We also analyzed the angular kyphosis at preoperative, and immediate and late post-operative periods. Results: There was an absolute weight gain in all patients with a mean gain of $8.7 \mathrm{~kg}$ (range 3 to $20 \mathrm{~kg}$ ). Six patients (75\%) gained weight and had increased value of the standard deviation, resulting in approximation of their weight in relation to the median considered normal weight versus age reference curve after starting treatment with VEPTR. Conclusion: The observed absolute weight gain was not accompanied by an improvement of nutritional status and there was no relationship between kyphosis correction and weight gain.
\end{abstract}

Keywords: Meningomyelocele; Prostheses and implants; Weight gain; Kyphosis; Spine.

\section{RESUMEN}

Objetivo: Evaluar la ganancia de peso y el cambio en el estado nutricional de los pacientes con mielomeningocele torácico después de usar la prótesis vertical expandible de titanio (VEPTR) como una opción para el tratamiento sin la fusión de la columna vertebral. Métodos: Estudio retrospectivo de ocho pacientes (edad media de 5,3 años, entre 3,6 y 7,6 años en el momento de la cirugía inicial), con mielomeningocele torácico que tenían escoliosis , medida por el método de Cobb, con valores superiores a $40^{\circ}$ y cifosis mayores de $80^{\circ}$, tratados con VEPTR en el Grupo de Escoliosis de la AACD en São Paulo. Se analizaron los pesos, las desviaciones estándar, en el que cada paciente se encontraba en la edad en comparación con tabla de edad versus peso de los valores de referencia de la OMS y también la cifosis angular en el preoperatorio y postoperatorio inmediato y tardío. Resultados: Hubo un aumento de peso absoluto en todos los pacientes con una media de 8,7 kg. (rango 30-20 Kg.). Seis pacientes (75\%) tuvieron un aumento de peso seguida de un aumento en el valor de la desviación estándar acercarse a la mediana de la curva edad versus peso referencia después del inicio del tratamiento con VEPTR .Conclusión: La ganancia de peso absoluto observada no fue acompañada por mejora del estado nutricional y no había ninguna relación entre la corrección de la cifosis y aumento de peso.

Descriptores: Meningomielocele; Prótesis e implantes; Aumento de peso; Cifosis; Columna vertebral.

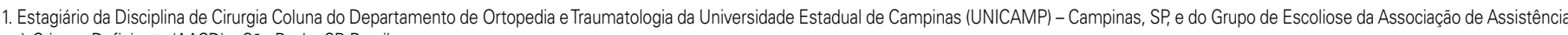
à Criança Deficiente (AACD) - São Paulo, SP, Brasil.

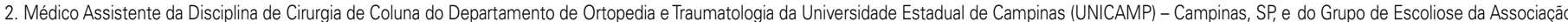
de Assistência à Criança Deficiente (AACD) - São Paulo, SP, Brasil.

3. Médico Assistente do Grupo de Escoliose da Associação de Assistência à Criança Deficiente (AACD) - São Paulo, SP, Brasil.

4. Médico Assistente da Disciplina de Cirugia de Coluna do Departamento de Ortopedia e Traumatologia da Universidade Estadual de Campinas (UNICAMP) - Campinas, SP, Brasil.

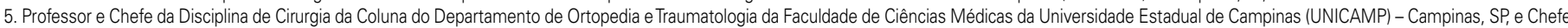
do Grupo de Escoliose da Associação de Assistência à Criança Deficiente (AACD) - São Paulo, SP, Brasil.
} 


\section{INTRODUÇÃO}

A incidência da mielomeningocele é de aproximadamente $1 / 2000$ nascidos vivos $(0.15 \%$ entre brancos e $0.04 \%$ entre afro-americanos), e, a cada ano, aproximadamente 6000 crianças nascem nos Estados Unidos com esta doença ${ }^{1}$. Nos pacientes com mielomeningocele podemos encontrar dois tipos de cifose: a paralítica por insuficiência muscular e a congênita ${ }^{2}$. A incidência de cifose nos portadores de mielomeningocele varia de 1 a $46 \%^{3,4} \mathrm{e}$ a forma congênita representa $13,9 \%{ }^{5}$. A cifose congênita (causada por falha da formação ou segmentação da vértebra), pode ser grave já ao nascimento com complicações relacionadas ao grande potencial de progressão, tendo média anual de $8,3^{\circ 6,7}$.

A compressão do conteúdo abdominal com concomitante aumento do esforço respiratório, o uso dos membros superiores e mãos para apoio do tronco, o sofrimento da pele com eventual ulceração na região proeminente e a dificuldade para sentar confortavelmente são alguns dos principais problemas relacionados com a cifose do segmento vertebral ${ }^{8-12}$. O baixo peso observado nestas crianças está relacionado ao comprometimento respiratório associado a problemas de deglutição e digestão que ocorrem pelo aumento da pressão do conteúdo abdominal contra o diafragma. A flexão aumentada do tronco também pode interferir na drenagem urinária, provocando infecções urinárias de repetição que deterioram a função renal ${ }^{13}$.

$\mathrm{O}$ aumento do volume do tórax e ganho de peso em pacientes portadores de síndrome da insuficiência torácica após o início do tratamento com o VEPTR (Vertical Expandable Prosthetic Titanium Rib) tem sido demonstrado na literatura ${ }^{14-16}$. O o objetivo do estudo é avaliar o ganho de peso nas crianças portadoras de mielomeningocele torácica submetidos ao tratamento com a prótese vertical expansível de titânio para costela (VEPTR).

\section{MÉTODOS}

Foi realizado estudo retrospectivo com oito pacientes portadores de mielomeningocele torácica e cifose congênita submetidos ao tratamento cirúrgico com VEPTR entre Outubro de 2006 e Julho de 2008. Todos os pacientes foram operados pelo Grupo de Escoliose da Associação de Assistência a Criança Deficiente (AACD) em São Paulo.

O tratamento com VEPTR foi indicado para as crianças com potencial de crescimento e/ou baixo peso para cirurgia de vertebrectomia e artrodese. Todos os procedimentos foram realizados em ambiente hospitalar com anestesia geral e em decúbito ventral.

As montagens foram feitas com duas hastes paramedianas (direita e esquerda), fixadas na costela e na pelve, por quatro incisões longitudinais: duas distais (paravertebral próximo ao ápice da crista ilíaca) e duas proximais (para escapular na altura da costela em que se realizaria a colocação dos ganchos). A montagem da porção distal do VEPTR necessitou descolamento da apófise da crista ilíaca para introdução do gancho no ilíaco e, da porção proximal, descolamento das partes moles, com preservação do periósteo da costela escoIhida. As duas hastes ficaram paralelas entre si e perpendiculares ao solo para evitar o desequilíbrio lateral durante o crescimento e/ ou alongamentos. A colocação do implante no plano submuscular e o fechamento por planos, buscando a maior cobertura com tecido mole, objetiva evitar saliência do implante ou ulcerações da pele. $\mathrm{O}$ uso de dreno de sucção não foi necessário em nenhum caso.

O pós-operatório foi realizado em Unidade de Terapia Intensiva (UTI) com fisioterapia respiratória, já nos procedimentos de alongamento não houve a necessidade de cuidados pós-operatórios em UTI. As crianças apresentavam exames laboratoriais sanguíneos normais (hemograma e coagulograma) e urocultura negativa na internação. Em todos os casos de montagem e alongamento realizou-se profilaxia antibiótica, de acordo com o padrão estabelecido pela Comissão de Controle de Infecção Hospitalar (CCIH). Todos apresentavam também teste de alergia ao látex. Os alongamentos, necessários para acompanhar o crescimento do tronco, são realizados por meio de incisões longitudinais ou transversas sobre as travas identificadas pela palpação. O período entre os alongamentos variou de quatro a seis meses.

Em nenhum caso, utilizou-se imobilização pós-operatória e o programa de reabilitação foi mantido, com orientação apenas para evitar atividades com flexão excessiva do tronco.

As variáveis clínicas analisadas foram: peso e as complicações decorrentes tanto do procedimento de montagem como de alongamento do sistema. Foram também comparadas as radiografias pré- e pós-operatória com avaliação do desvio no plano sagital (cifose), pelo método de Cobb.

Informações sobre idade e peso na época da cirurgia inicial e em todos os alongamentos foram obtidas. Em cada consulta de retorno, foram avaliadas as questões clínicas dos pacientes (peso, condições da pele e infecções locais ou em outros focos a distância) e obtidas radiografias de controle para a mensuração do ângulo da cifose pelo método de Cobb. Foram incluídos neste trabalho os pacientes com seguimento de pelo menos 22 meses.

O peso na época da cirurgia inicial e de cada internação para a realização do alongamento do VEPTR foi relacionado com a idade dos pacientes na ocasião e convertidos em desvios-padrão a partir da tabela idade versus peso para cada gênero da Organização Mundial de Saúde ${ }^{17,18}$

Os dados pré e pós-operatórios, imediatos e tardios, foram comparados para avaliar e o ganho de peso absoluto e a mudança nos valores do desvio-padrão destas crianças após o tratamento com VEPTR e se houve relação entre ganho de peso e diminuição do valor angular da cifose.

Foi utilizado o programa SPSS (Statistical Package for Social Sciences), em sua versão 19.0, para a obtenção dos resultados e adotamos o nível de significância de $5 \%(p<0,050)$, para a aplicação dos testes estatísticos.

\section{RESULTADOS}

Das oito crianças submetidas ao tratamento cirúrgico com VEPTR, quatro (50\%) eram meninas e quatro $(50 \%)$ meninos. A média da idade na cirurgia inicial foi de 5,3 anos (variando de 3,6 até 7,6 anos), todos os pacientes foram observados por no mínimo 22 meses com média de 29,1 meses (variando de 22 a 37 meses) (Tabela 1).

A média do peso pré-operatório foi de 13,4 kg (variando de 7 a $22 \mathrm{~kg}$ ) e a média do peso ao final do seguimento foi de $24 \mathrm{~kg}$ (variando de 10 a $42 \mathrm{~kg}$ ). Em todos os pacientes foi observado ganho de peso absoluto com média de $8,7 \mathrm{~kg}$ (variando de 3 a $20 \mathrm{~kg}$ ). A média do valor da cifose inicial, medida pelo método de Cobb foi de $119^{\circ}$ (variando de $86^{\circ}$ a $150^{\circ}$ ) e a média do valor da cifose no pós-operatório imediato foi de $84^{\circ}$ (variando de $69^{\circ}$ a $100^{\circ}$ ) levando a uma porcentagem de correção em media de $27,75 \%$ (variando de $2,3 \%$ a $46,4 \%$ ) (Tabela 2).

Todos os pacientes, com exceção de um, que possuía peso adequado para a idade, estavam em media 2,1 DP abaixo da mediana para idade (variando de -5 a $-0,2$ DP) antes da cirurgia de colocação do VEPTR. Desses 8 pacientes, 6 apresentaram ganho de peso e melhora no desvio padrão se traduzindo em aproximação de seu peso em relação à mediana considerada normal da curva

Tabela 1. Sexo, idade na cirurgia inicial, tempo de seguimento e número de alongamentos.

\begin{tabular}{c|c|c|c|c}
\hline Caso & Sexo & Idade & Seguimento & Alongamentos \\
\hline 1 & $M$ & $3 a 8 m$ & $29 m$ & 4 \\
\hline 2 & $F$ & $5 a 3 m$ & $34 m$ & 5 \\
\hline 3 & $F$ & $6 a 11 m$ & $37 m$ & 4 \\
\hline 4 & $M$ & $6 a 2 m$ & $25 m$ & 3 \\
\hline 5 & $M$ & $7 a 8 m$ & $35 m$ & 6 \\
\hline 6 & $F$ & $4 a 4 m$ & $25 m$ & 3 \\
\hline 7 & $F$ & $5 a 4 m$ & $22 m$ & 3 \\
\hline 8 & $M$ & $4 a 10 m$ & $22 m$ & 3 \\
\hline
\end{tabular}

M: masculino; F: feminino; a: anos; m: meses.

Tabela 2. Peso inicial, peso final, valor angular da cifose pré e pós-operatório.

\begin{tabular}{c|c|c|c|c}
\hline Caso & Peso inicial & Peso final & $\begin{array}{c}\text { Perfil cifose } \\
\text { pré-operatório * }\end{array}$ & $\begin{array}{c}\text { Perfil cifose } \\
\text { pós-operatório imediato* }\end{array}$ \\
\hline 1 & $11,8 \mathrm{~kg}$ & $17 \mathrm{~kg}$ & $108^{\circ}$ & $70^{\circ}$ \\
\hline 2 & $18 \mathrm{~kg}$ & $25 \mathrm{~kg}$ & $92^{\circ}$ & $69^{\circ}$ \\
\hline 3 & $22 \mathrm{~kg}$ & $42 \mathrm{~kg}$ & $125^{\circ}$ & $100^{\circ}$ \\
\hline 4 & $19 \mathrm{~kg}$ & $25 \mathrm{~kg}$ & $150^{\circ}$ & $92^{\circ}$ \\
\hline 5 & $17 \mathrm{~kg}$ & $34 \mathrm{~kg}$ & $130^{\circ}$ & $100^{\circ}$ \\
\hline 6 & $12,9 \mathrm{~kg}$ & $20 \mathrm{~kg}$ & $140^{\circ}$ & $75^{\circ}$ \\
\hline 7 & $13,7 \mathrm{~kg}$ & $21 \mathrm{~kg}$ & $118^{\circ}$ & $81^{\circ}$ \\
\hline 8 & $16,4 \mathrm{~kg}$ & $22 \mathrm{~kg}$ & $86^{\circ}$ & $84^{\circ}$ \\
\hline
\end{tabular}

Kg: quilogramas; *: valores em Graus medidos pelo método de Cobb. 
idade versus peso referência após o inicio do tratamento.

Ao final do seguimento os pacientes se apresentavam em média a - 0.9 DP (variando de -6 a $+2,5$ ) da mediana da curva idade versus peso referência. Um paciente, caso 1, apesar do ganho absoluto de peso, apresentou piora na curva idade versus peso e se encontrava ao final do seguimento com -0,2 DP abaixo de seu valor inicial.

Foi observado ganho absoluto de peso em todos os pacientes. Sete do total de $8(87,5 \%)$, inclusive o paciente que estava com peso adequado para idade, mostraram ganho de peso absoluto e melhora de suas posições na curva idade versus peso.

Aplicado o Teste de Friedman para avaliação das variáveis em questão, quanto ao ganho de peso absoluto, obtivemos um valor de $p<0,001$ e como tal diferença encontrada é estatisticamente significante, podemos afirmar que existe um efetivo crescimento dos valores de peso absoluto ao longo do tempo (Tabela 3).

Em relação à avaliação dos valores de desvio-padrão, apesar de haver uma clara tendência ao aumento dos mesmos, a melhora do status nutricional existe, mas não é estatisticamente significante, com valor de $\mathrm{p}=0,138$.

Analisando os valores pré e pós-operatórios imediatos da cifose e os correlacionando com o ganho de peso, não houve associação entre a porcentagem de correção da deformidade e o ganho de peso $(p=0,301)$

Dos oito pacientes do estudo, quatro (50\%) tiveram complicações relacionadas ao procedimento, sendo três infecções superficiais $(37,5 \%)$, uma delas acompanhada de soltura e uma $(12,5 \%)$ fratura de costela também acompanhada de soltura. Todas as complicações foram solucionadas com um único desbridamento/ reposicionamento sem repercuções significativas e não trouxeram perda ou atraso no ganho de peso.

Tabela 3. Aplicação do Teste de Friedman para análise do peso absoluto.

\begin{tabular}{c|c|c|c|c|c|c|c|c|c}
\hline $\begin{array}{c}\text { Bloco de } \\
\text { Variáveis }\end{array}$ & n & Média & $\begin{array}{c}\text { Desvio- } \\
\text { padrão }\end{array}$ & Mínimo & Máximo & $\begin{array}{c}\text { Percentil } \\
\mathbf{2 5}\end{array}$ & $\begin{array}{c}\text { Percentil 50 } \\
\text { (Mediana) }\end{array}$ & $\begin{array}{c}\text { Percentil } \\
\mathbf{7 5}\end{array}$ & $\begin{array}{c}\text { Significância } \\
\text { (p) }\end{array}$ \\
\hline peso 1 & 8 & 16,24 & 3,36 & 11,80 & 22,00 & 13,13 & 16,70 & 18,50 & \\
\hline peso 2 & 8 & 19,05 & 4,90 & 12,00 & 27,00 & 15,70 & 17,90 & 23,00 & \multirow{2}{*}{$<0,001$} \\
\hline peso 3 & 8 & 21,66 & 6,02 & 13,00 & 32,30 & 18,00 & 20,75 & 25,63 & \\
\hline peso 4 & 8 & 24,28 & 7,58 & 15,00 & 40,20 & 20,00 & 23,00 & 27,25 & \\
\hline
\end{tabular}

\section{DISCUSSÃO}

A avaliação do estado nutricional das crianças portadoras de mielomeningocele é essencial e deve fazer parte do cuidado multidisciplinar que estes pacientes demandam. A deformidade vertebral em cifose presente na mielomeningocele conduz complicações locais e sistêmicas. Ulcerações locais, incapacidade de sentar-se de maneira confortável e adequada, necessidade de usar os membros superiores e mãos para apoio, aumento da pressão intrabdominal com problemas de deglutição e digestão, drenagem urinária e deformidades torácicas complexas são alguns dos exemplos.

\section{REFERÊNCIAS}

1. Copp AJ. Neural tube defects. Trends Neurosci. 1993;16(10):381-3

2. Raycroft JF, Curtis BH. Spinal curvature in myelomeningocele: natural historyand etiology. In: American Academy of Orthopedic Surgeons Symposium on myelomeningocele. St. Louis: Mosby: 1972.

3. Burney DW Jr, Hamsa WR. Spina Bifida with myelomeningocele. Clin Orthop Relat Res. 1963;30:167-74.

4. Naik DR, Lendon RG, Barson AJ. A radiological study of vertebral and rib malformations in children with myelomeningocele. Clin Radiol. 1978;29(4):427-30

5. Carstens $\mathrm{C}$, Koch H, Brocai DR, Niethard FU. Development of pathological lumbar kyphosis in myelomeningocele. J Bone Joint Surg Br. 1996:78(6):945-50.

6. Banta JV, Hamada JS. Natural history of the kyphotic deformity in myelomeningocele. J Bone Joint Surg Am. 1976;58:279.

7. Hoppenfeld S. Congenital kyphosis in myelomeningocele. J Bone Joint Surg Br. 1967:49(2):276-80.

8. Christofersen MR, Brooks AL. Excision and wire fixation of rigid myelomeningocele kyphosis. J Pediatr Orthop. 1985;5(6):691-6.

9. Hall JE, Poitras B. The management of kyphosis in patients with myelomeningocele. Clin Orthop Relat Res. 1977;(128):33-40.

10. Heydemann JS, Gillespie R. Management of myelomeningocele kyphosis in the older child by kyphectomy and segmental spinal instrum

11. Lindseth RE. Spine deformity in myelomeningocele. Instr Course Lect. 1991;40:273-9

12. McMaster MJ. The long-term results of kyphectomy and spinal stabilization in children
O objetivo do tratamento é o controle da curva por meio da artrodese. Tal controle é obtido pelo restabelecimento completo do equilíbrio sagital, estabilizando a coluna sobre a bacia, garantindo, assim, a durabilidade da correção. A idade ideal para o tratamento cirúrgico, o método de correção da deformidade, a extensão e o tipo da instrumentação são pontos ainda a serem definidos.

Vitale et al. ${ }^{19}$ tiveram uma taxa de $30 \%$ de complicações pós-operatórias com esse sistema, sendo infecção a mais frequente $(13,5 \%)$, seguida de soltura do implante $(8,1 \%)$, associação entre infecção e soltura $(5,4 \%)$ e complicações dermatológicas $(2,7 \%)$ em pacientes com fusão de costelas, hipoplasia torácica ou deformidades progressivas. Nos portadores de deformidades neuromusculares, a taxa relatada de complicação é de $23,5 \%{ }^{20}$.

A prótese vertical expansível de titânio para costela (VEPTR) é uma opção eficaz no tratamento das deformidades vertebrais ou torácicas neste grupo de pacientes levando ao aumento do tamanho do tórax e melhora da posição e equilíbrio do tronco ${ }^{14,15}$. Para que estas crianças não apresentem complicações pós operatórias como deiscência de sutura com ulceração e exposição do material de implante, já que serão submetidas a vários procedimentos, ou atelectasias com conseqüente insuficiência respiratória e necessidade de assistência ventilatória é desejavel que as mesmas estejam adequadamente nutridas.

A utilização do VEPTR nos pacientes portadores de mielomeningocele torácica com cifose congênita permite correção da cifose e do equilíbrio sagital em idade mais precoce, permitindo com isso desenvolvimento das habilidades manuais pela liberação dos membros superiores ao sentar e ganho de peso pelo aumento do volume abdominal.

Na literatura não há uma curva para acompanhamento nutricional específica para esse tipo de pacientes o que nos leva a usarmos os padrões para crianças saudáveis.

Nosso estudo demonstrou que em $100 \%$ dos casos analisados houve ganho ponderal absoluto e que em $87,5 \%$ dos casos este ganho se traduziu também em aumento nos valores de desvio-padrão na curva idade versus peso demonstrando uma melhora no status nutricional, apesar de não estatisticamente significante, dos pacientes com o passar do tempo. Não houve relação entre a correção da cifose e o ganho de peso.

\section{CONCLUSÃO}

Foi observado que há ganho absoluto de peso estatisticamente significativo. Esperávamos que este ganho ponderal se refletisse numa melhora do status nutricional com aumento dos valores do desvio-padrão na curva idade versus peso. Observamos melhora do status nutricional mas que no entanto não foi estatisticamente significativa, talvez porque na verdade estamos comparando grupos diferentes de indivíduos, crianças saudáveis versus crianças portadoras de mielomeningocele, logo, é necessário o desenvolvimento de curvas de acompanhamento nutricional específicas para este grupo de pacientes. with myelomeningocele. Spine (Phila Pa 1976). 1988;13(4):417-24

13. Zuiani GR, Cavali PTM, Santos MAM, Rossato AJ, Lehoczki MA, Risso Neto MI, et al. Uso da prótese vertical expansível de titânio para costela no tratamento da cifose congênita em portadores de mielomeningocele torácica Coluna/Columna;8(3):286-96.

14. Campbell RM Jr, Hell-Vocke AK. Growth of the thoracic spine in congenital scoliosis after expansion thoracoplasty. J Bone Joint Surg Am. 2003;85(3):409-20.

15. Motoyama EK, Deeney VF, Fine GF, Yang Cl, Mutich RL, Walczak SA, et al. Effects on lung function of multiple expansion thoracoplasty in children with thoracic insufficiency syndrome: a longitudinal study. Spine (Phila Pa 1976). 2006;31(3):284-90.

16. Skaggs DL, Sankar WN, Albrektson J, Wren TA, Campbell RM. Weight gain following vertical expandable prosthetic titanium ribs surgery in children with thoracic insufficiency syndrome. Spine (Phila Pa 1976). 2009;34(23):2530-3.

17. WHO Child Growth Standards: Length/height-for-age, weight-for-age, weight-for-length, weight-for-height and body mass index-for-age: methods and development. Geneva: World Health Organization; 2006.

18. WHO Growth reference data for 15-19 years, 2007. Disponivel em: http://who int/growthref/en/

19. Vitale MG, Matsumoto H, Roye DP Jr, Gomez JA, Betz RR, Emans JB, et al. Health-related quality of life in children with thoracic insufficiency syndrome. J Pediatr Orthop. 2008;28(2):239-43.

20. Landim E, Cavali PTM, Santos MAM, Pasqualini W, Boechat RCB, Andrade SMS. Uso da prótese vertical expansível de titânio para costela (VEPTR) com opção na instrumentação sem fusão para tratamento da escoliose neuromuscular. Coluna/Columna. 2008;7(2):160-6. 